\title{
A smooth tracking algorithm for capacitive touch panels
}

\author{
Zu-Cheng Zhang, Ju-Peng Li and Mo-Yu Li \\ School of Electronic Information Engineering, Beijing Jiaotong University, Beijing, \\ China \\ E-mail: lijupeng@bjtu.edu.cn
}

\begin{abstract}
Capacitive touch panels (CTPs) have been more popular owing to its excellent interactive performance. However, electronic magnetic interference, display noise and process variation negatively influence the accuracy of predictions of touch positions. A smooth tracking algorithm for capacitance touch panels based on Kalman filter was proposed in this paper. Original touch capacitance data and touch noise characteristic were detected using a micro-controller unit and a sensor integrated circuit. Additionally, the noise of touch trajectory was suppressed by Kalman algorithm timely through process model and measurement model of touch trajectory smooth system. Experimental results indicated that the proposed touch trajectory smooth algorithm has better performance compared to the moving average filtering and wavelet filtering algorithm, and the algorithm can be used to capacitive touch panel systems.

Keywords: Capacitive Touch Panel; Touch Signal Noise; Kalman Filter; Tracking
\end{abstract} Smoothness.

\section{Introduction}

The capacitive touch panels have permeated into our life and accepted by consumers because of its excellent performance. But at the same time, they are easily influenced by the noise inside the equipment and produce fake and wrong response[1] [2]. Touch driver need to be the same level of user experience, for the noises of $\mathrm{AD} / \mathrm{DC}$ electronic systems internal, display driver, antenna or other noises coming from other sources[3] [4], then it is extremely important to take the necessary and feasible filter algorithm to reduce noise for capacitive touch screen and improve the localization accuracy. Although H. Sanghyun et al. put forward touch control circuit based on differential detection method to reduce prediction error and improve the dynamic range of sensing voltage, but it is more difficult to use hardware to reduce the noise than software algorithm [3] [5], so the Moving Average filtering (MAF), Wavelet transform et al. is often used to eliminate the measurement noise and predict touch position [6] [8]. F.Golnaraghi et al. predicted position and velocity in the hybrid system based on 
position detection and inertial measurement unit[9] [10], the solution of these problems provides a thought in trajectory smooth of capacitive touch panels processing for this article.

\section{Analysis of Capacitive Touch Signal and Noise Characteristics}

It need to find the maximum value of capacitance for per frame, the value is usually set as $P_{\max }(k)=\left(P_{\max x}(k), P_{\operatorname{maxy}}(k)\right)$, when the maximum value is bigger than the threshold $T$ we set based on hardware system, it will judge the touch events occur. The touch point coordinates can be weighted calculated from the original capacitance data which's center is $P_{\max }(k)$ in the field of $\Omega$. Then the coordinates will be set as $P(k)=\left(P_{x}(k), P_{y}(k)\right)$,

$$
P_{x}(k)=\frac{\sum_{x \in \Omega} \sum_{y \in \Omega} C_{x y} *\left(P_{\max . x}(k)+x\right)}{\sum_{x \in \Omega} \sum_{y \in \Omega} C_{x y}}, P_{y}(k)=\frac{\sum_{x \in \Omega} \sum_{y \in \Omega} C_{x y} *\left(P_{\max y}(k)+y\right)}{\sum_{x \in \Omega} \sum_{y \in \Omega} C_{x y}}
$$

The coordinates of point are lined up with the time $\mathrm{k}$, which are obtained from a full touch process. The CTP track signal $P(k)(k=1, \ldots, N)$ can be composed from the coordinates.

Figure 1(a) is the starting position of the touch, the noise is caused by the finger trembling; figure $1(\mathrm{~b})$ is the " $\mathrm{Z}$ " shaped curve, the curve distributes in the whole trajectory uniformly. The location of curve line of this part is corresponding to the driving electrode of $\mathrm{X}$ axis, and it can be assumed this kind of noise is mainly caused by the uneven distribution of the diamond electrode; figure $1(\mathrm{c})$ is the flat trajectory curve, and slight fluctuations are formed by a variety of external interference effect.

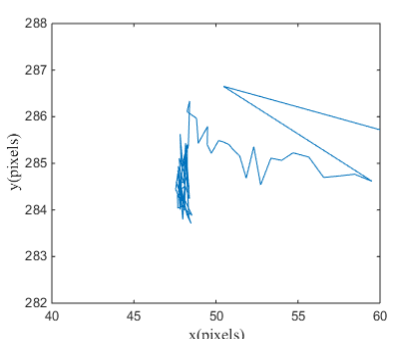

(a)

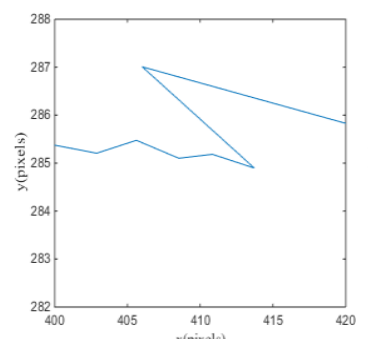

(b)

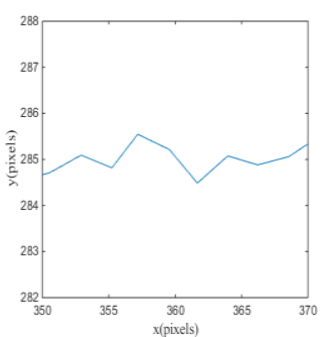

(c)

Fig. 1 Analysis of several common noise trajectory characteristics: (a) Finger trembling noise; (b) "Z" shaped trajectory noise; (c) Flat trajectory noise

\section{Touch Trajectory Smoothing Algorithm}

The Kalman filtering is a kind of time domain recursive algorithm, which is widely used in navigation, target location and tracking, and so on, as one of the most important optimal estimation theory [9] [10]. For the problem of capacitive 
touch screen trajectory smoothing, it can assume the velocity of finger was uniform on the capacitive touch panel, and the state space model can be established:

State equation:

$$
M(k)=\boldsymbol{A} M(k-1)+W(k)
$$

\section{Measurement equation}

$$
P(k)=\boldsymbol{H} M(k)+V(k)
$$

Where

$$
M(k)=\left[\begin{array}{c}
P_{x}(k) \\
v_{x}(k) \\
P_{y}(k) \\
v_{y}(k)
\end{array}\right], A=\left[\begin{array}{cccc}
1 & T_{0} & 0 & 0 \\
0 & 1 & 0 & 0 \\
0 & 0 & 1 & T_{0} \\
0 & 0 & 0 & 1
\end{array}\right], P(k)=\left[\begin{array}{l}
P_{x}(k) \\
P_{y}(k)
\end{array}\right], H=\left[\begin{array}{cccc}
1 & 0 & 0 & 0 \\
0 & 0 & 1 & 0
\end{array}\right]
$$

$M(k)$ : contains process state vector position and velocity of $x, y$ coordinate axes; $A$ : system state transition matrix; $P(k)$ : update the measurement position vector; $H$ : system observation matrix; $W(k)$ and $V(k)$ : zero-mean Gaussian white sequences with a zero cross correlation with each other, variance is $Q$ and $R$ respectively.

In order to facilitate the trajectory parameters, the touch trajectory is selected as parallel to the $x$ or $y$ axis, so as to fix the $y$ direction of the coordinates. And the trajectory can be get along the $x$ direction, $P_{y}(k)$ can be expressed as:

$$
P_{y}(k)=Y_{0}+n_{y}(k)
$$

$\mathrm{Y} 0$ is the expectation of the touch signal in the $\mathrm{y}$ direction, ny $(\mathrm{k})$ is the noise for the $\mathrm{k}$ time on the $\mathrm{y}$ direction, in general $\mathrm{nx}(\mathrm{k})=\mathrm{ny}(\mathrm{k})=\mathrm{n}(\mathrm{k})$.

\section{Test and Analysis of Algorithm Performance}

The experiment acquisition system is made of a 7 inch capacitive touch screen which has $20 \times 12$ ITO diamond electrode. In order to measure the affection of the touch trajectory smoothing filter method proposed in the paper, the MAF filter and Wavelet transform filter are presented to compare and analysis .

\subsection{Qualitative analysis of 3 typical trajectory noise smoothing filters}

Touch trajectory includes 3 typical trajectory noises, figure 2 shows the filter results of 3 typical trajectory noise. 

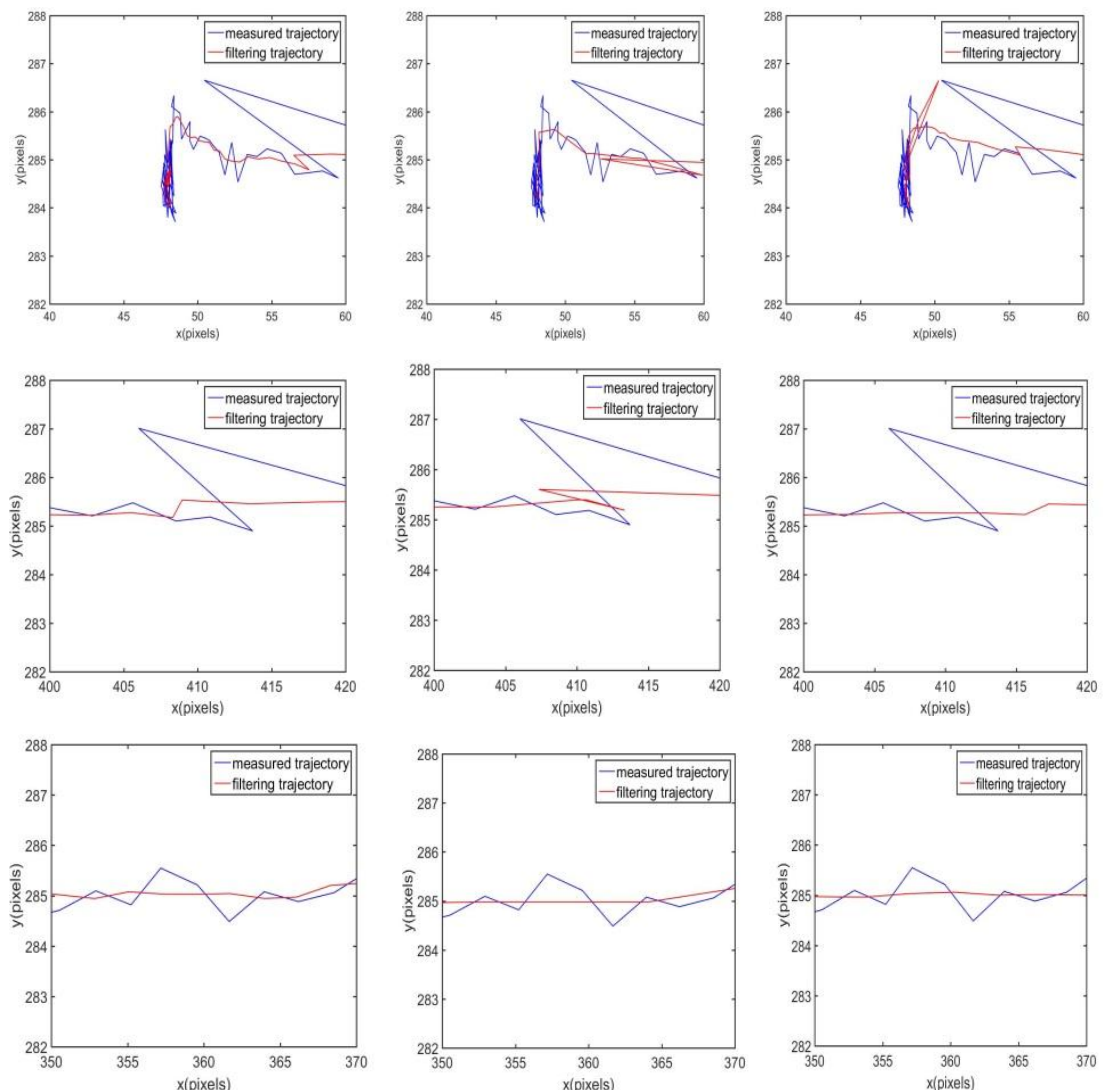

(a)

(b)

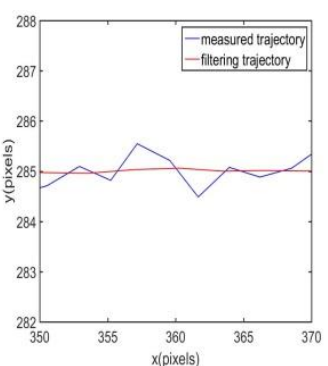

(c)

Fig. 2 Three typical noises filter results of different algorithms: (a) MAF smoothing results; (b) Wavelet smoothing results; (c) the proposed algorithm smoothing results

The first line in Fig. 2 is finger jitter noise, it is obvious the proposed filtering algorithm in this paper jump out with minimal fold lines, and after the jump with the smoother curve moving to next line; the second line is the "Z" trajectory, and wavelet transform algorithm has the worst results, because there are still obvious "Z" trajectory, only the bending amplitude has a slightly decrease, while the proposed algorithm has achieved the best results of smoothing, which will also be reflected in the back of the quantitative analysis; the third line is the flat part, three smoothing algorithms all can eliminate the noise effectively, the results of MAF algorithm have low frequency interference, wavelet transform algorithm has upturned phenomenon in tail, the proposed smoothing algorithm is close to the ideal linear touch trajectory. 


\subsection{Quantitative analysis of the result of smoothing filter}

In order to quantitatively measure the performance of the different algorithms in the touch trajectory, the average smoothness of the trajectory was calculated.

The experiment collected 10 (T1 T10) trajectory signals from the capacitive touch screen data in randomly in order to compare the performance for three filter method. The results of noise suppression has shown in Table 1.

Tab. 1 the trajectory smoothness of three filter method and consumption time (seconds)

\begin{tabular}{lllll}
\hline Savg & Raw data & MAF & Wavelet & Our algorithm \\
\hline T1 & 2.8211 & $1.1513 / 0.0028$ & $4.2714 / 0.1185$ & $0.3159 / 0.0049$ \\
T2 & 1.6202 & $0.4837 / 0.0096$ & $2.4252 / 0.2911$ & $0.1322 / 0.0114$ \\
T3 & 2.0209 & $0.6081 / 0.0048$ & $2.6716 / 0.2173$ & $0.2036 / 0.0071$ \\
T4 & 1.5286 & $0.4659 / 0.0102$ & $2.2643 / 0.3823$ & $0.1440 / 0.0145$ \\
T5 & 1.5667 & $0.5557 / 0.0059$ & $2.5727 / 0.2003$ & $0.1768 / 0.0084$ \\
T6 & 2.9968 & $1.0294 / 0.0029$ & $4.8427 / 0.1147$ & $0.3816 / 0.0046$ \\
T7 & 1.2842 & $0.4190 / 0.0049$ & $2.9810 / 0.1826$ & $0.1368 / 0.0066$ \\
T8 & 1.1489 & $0.3905 / 0.0059$ & $2.7265 / 0.1991$ & $0.1290 / 0.0071$ \\
T9 & 1.2371 & $0.4342 / 0.0049$ & $3.1694 / 0.2223$ & $0.1374 / 0.0071$ \\
T10 & 1.9440 & $0.7652 / 0.0064$ & $4.1793 / 0.1384$ & $0.2242 / 0.0103$ \\
\hline
\end{tabular}

From the definition of touch trajectory average smoothing, average smoothing parameter Savg is smaller, which represents the touch trajectory is more smooth. For the same touch data, the average smoothing parameter Savg is smaller, which represents the algorithm is better to noise suppression. Table 1 gives the calculation time and three trajectory average smoothness Savg. The experimental results demonstrate: though the Kalman algorithm increases computation time of compared to the MAF algorithm with a small quantity, the time consumption level do not change. It demonstrates that Kalman filter can meet the requirements of the bottom driver. The wavelet transform algorithm cost the most time.

\section{Conclusion}

Though the touch signal is easily affected by variety noises of interference sources, it can get a more smooth touch trajectory through the appropriate processing method, which is the basis for a good touch experience. In this paper, a touch trajectory smoothing algorithm based on Kalman filtering is proposed through the analysis of the characteristics of trajectory noise. The experiment collects many touch trajectory data, after comparing the performance of the Kalman algorithm with MAF filtering and Wavelet transform filtering, it come to conclusion that the three methods of trajectory filter can all make the original 
trajectory more smooth and reduce the unstable measurement noise, the proposed smoothing algorithm in this paper has obtained the best smooth trajectory and is suitable for applications in CTP systems.

\section{References}

1. P. T. Krein, R. D. Meadows, et al.. The electroquasistatics of the capacitive touch panel [J]. IEEE Transactions on Industry Applications, 1990, 26 (3): 529-534.

2. G. Shuo and M. David. Reduction of noise spikes in touch screen systems by low pass spatial filtering [J]. IEEE Journal of Display Technology, 2016, 12 (9): 957-963.

3. H. Sanghyun, M. Hyunggun, et al.. $72 \mathrm{~dB}$ SNR, $240 \mathrm{~Hz}$ frame rate readout IC with differential continuous-mode parallel architecture for larger touchscreen panel applications [J]. IEEE Transactions on Circuits and systems, 2016, 63 (7): 960-971.

4. C.L.Lin, et al.. Pressure sensitive stylus and algorithm for touch screen panel [J]. IEEE/OSA Journal of Display Technology, 2013, 9(1): 17-23.

5. J. S. Lee, D. H. Yeo, et al.. An LCD-VCOM-Noise Resilient MutualCapacitive Touch-Sensor IC Chip With a Low-Voltage Driving Signal [J]. IEEE Sensors Journal, 2015, 15 (8): 4595-4602.

6. ZHAN Siwei, WEI Yancun, LI Bo, and LIU Wei. Design of control chip for projection type capacitive touch screen[J]. Microelectronics, 2013, 43(3): 365-368.

7. D. H. Yeo, S. H. Kim, et al.. A SNR-enhanced mutual-capacitive touchsensor ROIC using an averaging with three specific TX frequencies, a noise memory, and a compact delay compensation circuit [J]. IEEE Sensors Journal, 2016, 16 (18): 6931-6938.

8. B. Li, T. C. Wei, et al.. A touch prediction and window sensing strategy for low-power and low-cost capacitive multi-touch screen systems [J]. IEEE Journal of Display Technology, 2016, 12 (6): 646-657.

9. S. P. Won, W. W. Melek, and F. Golnaraghi. A Kalman/Particle Filterbased position and orientation estimation method using a position sensor/inertial measurement unit hybrid system [J]. IEEE Transactions on Industrial Electronics, 2010, 57 (5): 1787-1798.

10. T. H. S. Li, Y. T. Su, et al.. Dynamicbalance control for biped robot walking using sensor fusion, Kalman filter, fuzzy logic. IEEE Transactions Industrial Electron, 2012, 59(11), 4394-4408. 